\title{
Analyzing Key Factors of Human Resources Management
}

\author{
Lourdes Sáiz ${ }^{1}$, Arturo Pérez ${ }^{2}$, Álvaro Herrero ${ }^{1}$ and Emilio Corchado ${ }^{3}$ \\ ${ }^{1}$ Department of Civil Engineering, University of Burgos \\ C/ Francisco de Vitoria s/n, 09006 Burgos, Spain \\ ${ }^{2}$ Investigador Programa Torres Quevedo, TTT Diseño, Comunicación y Contenidos, S.L. \\ Reyes Católicos, 41, 1, 09005 Burgos, Spain \\ ${ }^{3}$ Departamento de Informática y Automática, Universidad de Salamanca \\ Plaza de la Merced, s/n, 37008 Salamanca, Spain \\ \{lsaiz,ahcosio\}@ubu.es, arrturo@hotmail.com, escorchado@usal.es
}

\begin{abstract}
This study presents the application of an unsupervised neural projection model for the analysis of Human Resources (HR) from a Knowledge Management (KM) standpoint. This work examines the critical role that the acquisition and retention of specialized employees play in Hi-tech companies, particularly following the configuration approach of Strategic HR Management. From the projections obtained through the connectionist models, experts in the field may extract conclusions related to some key factors of the HR Management. One of the main goals is to deploy improvement and efficiency actions in the implantation and execution of the HR practices in firms. The proposal is validated by means of an empirical study on a real case study related to the Spanish Hi-tech sector.
\end{abstract}

Keywords: Unsupervised Neural Networks, Knowledge Management, Human Resources Management, Acquisition \& Retention.

\section{Introduction}

Knowledge Management (KM) enables organizations to capture, share, and apply the collective experience and the know-how (knowledge) of their staff. For KM to be successfully applied in organizations, it is necessary to develop and implement knowledge infrastructures [1]. These knowledge infrastructures consist of three main dimensions: people, organizational and technological systems.

In recent years, the deployment of information technology has become a crucial tool for enterprises to achieve a competitive advantage and organizational innovation [2]. In keeping with this idea, Artificial Intelligence (AI) [3] can be applied in KM systems in order to speed up processes, classify unstructured data formats that KM is unable to organize, visualize the intrinsic structure of data sets, and select employeerelated knowledge from large amounts of data, among other processes. 
In keeping with this idea, the present study deals with Human Resource (HR) Management in Hi-tech companies that allows improving the interpretation and processing of the information related to HR practices in firms. In a more precise way, this work is intended for detecting situations of effective and ineffective management and diagnosing the most advisable actions for every case.

To identify the position of a company according to its HR Management, neural projection models are applied in this research. The main goal is to identify the status of a company to perform subsequent corrective actions under the frame of a Hybrid Artificial Intelligence System, as proposed in [4]. This is an ongoing research that started with a wider analysis of HR features [5] and is now focused on the acquisition and retention settings related with $\mathrm{R}+\mathrm{D}$ employees of high-tech firms. These are key issues as there is a lack of this kind of employees, according to the data provided by the Spanish National Institute of Statistics [6]. Decisions in this field are strongly related with rivalry. In this sense, KM provides us with valuable information to determine the most effective politics on HR acquisition and retention. This allows proposing to each firm the decision to be taken in order to improve an unsatisfactory or inefficient HR situation.

The study is structured in the following way. Section 2 introduces the field of HR and $\mathrm{KM}$, while section 3 describes the unsupervised neural projection model applied in this research. Section 4 covers the application to a real-world problem and the experimental study that has been carried out. Finally, Section 5 presents the conclusions and some proposals for future work.

\section{Knowledge Management and Human Resources}

In the present economic context, similar changes have occurred in almost every economic sector, cutting down the strategic importance of tangible resources. The more knowledge demanding a sector is (as the case of high technology), the more important knowledge-based resources are as value generators. Organizations investing in innovation obtain more benefits than those who do not [7].

Innovation is an important result for firms, mainly in technological industries [8]. Furthermore, in global and dynamic environments, the successful firms do need an explicit strategy involving innovation and KM for fast adaptation [9]. The main reason for this is that the introduction of new products and services depends on the capability to generate, combine and exchange the new knowledge [10]. Thus, the source of innovation is the integration of new knowledge with the knowledge previously stored by individuals in a specialized form [11].

Organizations may achieve good performance results if they are able to both take advantage of the knowledge held by a group of people and to effectively organize them [12]. The processes of knowledge creation, transformation, integration and influence are related with the internal collaboration of employees. HR Management promotes and eases knowledge gathering, and at the same time it leads to implement the previously mentioned processes. It is especially critical when HR are strongly linked to technological resources. 
One of the ways to achieve the required level of HR is by employing those people who have previous experience and specialized knowledge. In keeping with this idea, HR planning, extensive recruiting, and selection practices are key factors to achieve a high level of HR. However, there is a lack of such employees and, as a result, there is a serious rivalry between firms. Thus, the firms that will get the employees with high knowledge level would be those applying exhaustive recruiting policies such as multiple selection sources, a wide group of candidates, high salaries, among others.

Employing specialized staff will only partially solve the associated problem because, at the same time, these HR may move away to a different firm. As a firm generates value through their employees, success partially relies on its ability to keep them [12]. As knowledge and knowledge creation take place in the individuals, it is a menace that abilities and specific knowledge may disappear, reducing the firm capability to generate new knowledge.

It is important for knowledge acquisition that employees know who do they have to contact in order to find the required knowledge [13]. Hence, personnel stability is essential to perfect development of knowledge resources [14]. The stay of such employees may be guaranteed through HR management systems including compensations and rewards higher than average, comfortable working places, pleased and committed employees, social training, employee-organization connection, welcoming politics, labour safety, autonomy, participating culture, etc.

Taking into account the above mentioned ideas, a wide research on analysis factor was carried out [5] ranging from starting advanced HR practices to explaining firm results. To do so, it focused on intermediate related indicators such as employee characteristics, organizational capabilities and some other internal features. The studied factors were: five settings for HR practices (acquisition, development, compromise, retention and flexibility), five features of employees (human, social and organizational capital, motivation and turn over), four organization capabilities (knowledge creation and application, organizational flexibility and information technologies) and some other internal features (strategic vision, HR emphasis, heterogeneity, and task-associated technology). From the above mentioned, the present study has only taken into account those decisions related to the acquisition of specialized personnel, employees retention and the configuration of these two ones (Strategic HR Management [15]). The remaining factors will be covered in future work. Some HR practices are coherent with the strategic objectives and greatly contribute to the achievement of firm results. Some of the features related with these practices have been considered in the present study to visually identify the situation of hi-tech firms according to their HR situation.

\section{Neural Projection Model}

Projection models perform changes to the spatial coordinates of high-dimensional data in order to project them onto a lower dimensional space. The main goal is to identify the patterns that exist across dimensional boundaries by identifying "interesting" directions in terms of any specific index or projection. Such indexes or projections are, for example, based on the identification of directions that account for 
the largest variance of a data set -as is the case of Principal Component Analysis (PCA) [16], [17]- or the identification of higher order statistics such as the skew or kurtosis index -as is the case of Exploratory Projection Pursuit (EPP) [18]. Having identified the most interesting projections, the data is then projected onto a lower dimensional subspace plotted in two or three dimensions, which makes it possible to examine its structure with the naked eye.

The combination of projection techniques together with the use of scatter plot matrices is a very useful visualization tool to investigate the intrinsic structure of multidimensional data sets, allowing experts to study the relations between different components, factors or projections, depending on the applied technique.

The solution proposed in this research applies an unsupervised neural model called Cooperative Maximum Likelihood Hebbian Learning (CMLHL) [19]. It is based on Maximum Likelihood Hebbian Learning (MLHL) [19], and introduces the application of lateral connections [20], [21] derived from the Rectified Gaussian Distribution [22]. This connectionist model has been chosen because it reduces the data dimensionality while preserving the topology in the original data set. Considering an $\mathrm{N}$-dimensional input vector $(x)$, and an M-dimensional output vector $(y)$, with $W_{i j}$ being the weight (linking input $j$ to output $i$ ), then CMLHL can be expressed as:

1. Feed-forward step:

$$
y_{i}=\sum_{j=1}^{\mathrm{N}} W_{i j} x_{j}, \forall i
$$

2. Lateral activation passing:

$$
y_{i}(t+1)=\left[y_{i}(t)+\tau(b-A y)\right]^{+} .
$$

3. Feedback step:

4. Weight change:

$$
e_{j}=x_{j}-\sum_{i=1}^{M} W_{i j} y_{i}, \forall j
$$

$$
\Delta W_{i j}=\eta \cdot y_{i} \cdot \operatorname{sign}\left(e_{j}\right)\left|e_{j}\right|^{p-1} .
$$

Where: $\eta$ is the learning rate, $\tau$ is the "strength" of the lateral connections, $b$ the bias parameter, $p$ a parameter related to the energy function [19], [21] and $A$ a symmetric matrix used to modify the response to the data [21]. The effect of this matrix is based on the relation between the distances separating the output neurons.

\section{Experimental Study}

To empirically validate the importance of the acquisition and retention factors, this study has covered 126 Spanish organizations related with high-technology. 267 R+D employees from these firms were surveyed in order to analyze the HR strategies and subsequently improve the status of the analyzed firms. 
The average profile of these hi-tech firms is that of an organization with 266 employees, manufacturing products and services (111 out of the 126 studied organizations). $47 \%$ of the analyzed firms claim they innovate in both products and services, running $124 \mathrm{R}+\mathrm{D}$ annual programs. $44 \%$ of the analyzed firms are members of a corporate group, $16 \%$ of them being international.

As only the HR acquisition and retention factors are considered in the present study, features related with these two factors were analyzed from the surveyed data. These features are described in the following table.

Table 1. Analyzed features for both acquisition and retention factors.

\begin{tabular}{|c|c|}
\hline Acquisition factor & Retention factor \\
\hline $\begin{array}{l}\text { 1.- There is a plan to find the required } \\
\text { HR abilities. }\end{array}$ & $\begin{array}{l}\text { 1.- Candidates are selected according to } \\
\text { their fitting with the firm. }\end{array}$ \\
\hline $\begin{array}{l}\text { 2.- A wide group of candidates is } \\
\text { considered for vacancies. }\end{array}$ & $\begin{array}{l}\text { 2.- Employees match with the } \\
\text { organization culture. }\end{array}$ \\
\hline $\begin{array}{l}\text { 3.- Initial salaries are higher than } \\
\text { competitors to attract candidates. }\end{array}$ & 3.- New employees are supported. \\
\hline $\begin{array}{l}\text { 4.- A high amount of money is spent to } \\
\text { contract the right person. }\end{array}$ & $\begin{array}{l}\text { 4.- Social and outdoor activities are } \\
\text { sponsored by the firm for employees } \\
\text { to know each other. }\end{array}$ \\
\hline $\begin{array}{l}\text { 5.- Trainers of new employees are } \\
\text { carefully chosen and prepared. }\end{array}$ & $\begin{array}{l}\text { 5.- Higher salaries than competitors are } \\
\text { offered to keep employees. }\end{array}$ \\
\hline \multicolumn{2}{|c|}{$\begin{array}{l}\text { Five features have been used to define each factor. The values for all these features } \\
\text { e discrete ones and range from } 1 \text { (strongly low) to } 5 \text { (strongly high). As a result, five } \\
\text { atures from each firm (126) have been gathered in both dataset (acquisition and } \\
\text { tention). }\end{array}$} \\
\hline
\end{tabular}

\subsection{Results}

This section comprises an analysis of the best projections obtained in the abovedescribed experimental study by applying the CMLHL model to the data related with the two HR factors. The visualized groups in each one of the projections has been labelled $(1.1,1.2 \ldots)$ in Fig. 1 and Fig. 2 for easy referencing.

\section{Acquisition Factor}

Firstly, CMLHL was applied to the acquisition features, generating the projection in Fig. 1. An in-deep analysis of this projection allows us to define the common characteristics for each one of the clearly identified groups (labelled in Fig. 1) according to the dataset features.

Considering the first feature for the acquisition factor ("There is a plan to find the required HR abilities”), the firms are decreasingly ordered from left to right in Fig. 1. Thus, firms in the left third of the projection (groups 1.1, 1.2, 2.1, 2.2, 2.3, and 2.4) are those with the highest values for this feature, which means that these firms have designed and applied the required abilities for the economic activity. Those firms in 
the middle of the projection identified this ability plan as incomplete and it is even worse in the case of companies in the right third of Fig. 1 (groups 4.1, 4.2, 4.3, 4.4, 4.5, 5.1, 5.2, and 5.3). According to this result, it has been checked that most of the firms have not completed the staff ability plan that is required and critical in order to fulfil the criteria for the acquisition factor.

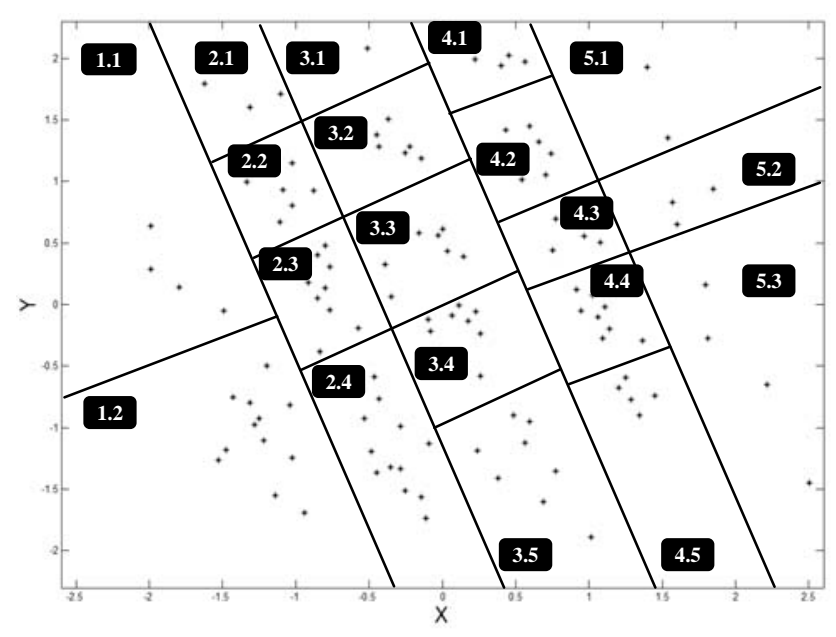

Fig. 1. CMLHL projection of the Acquisition Factor dataset.

Regarding the second feature for the acquisition factor, the CMLHL projection provides a clearer clustering of firms. The firms are horizontally ordered, taking the highest values for this feature in the left side of the projection and the lowest values in the right side of the projection. Most of the companies are in an intermediate location, twelve of the companies take the highest value for this feature (Groups 1.1 and 1.2 in Fig. 1) while nine of them take the lowest value (Groups 5.1, 5.2 and 5.3 in Fig. 1). The more precisely the ability requirements are defined, more candidates will apply for the vacancies.

The third feature of the acquisition factor reveals a vertical ordering of the data in Fig. 1. The firms at the bottom of the CMLHL projection are those taking the highest values for this feature, while the ones at the top of the projection take the lowest value. After the visual analysis it can be concluded that many of the firms take high values regarding this feature, so they have the best employees from the market.

The fourth feature shows a vertical ordering of the data in Fig. 1, as in the case of the previous one. It is coherent with the previous feature as those firms that care about the knowledge of beginner trainers do also pay higher initial salaries than competitors.

The fifth feature complements the previous ones in the study of acquisition factor. The grouping obtained through CMLHL (Fig. 1) is not as precise as previously indicated. However, there is a kind of ordering, as those firms in a better situation are in the left side of the projection and those in the worse situation are in the right side.

From a general perspective, the projection organizes the firms in nineteen different groups (from 1.1 to 5.3). This global order leads to gather in group 1.2 those firms with the best policies regarding HR. On the other hand worst HR policies cause firms 
to be located in groups 5.1 and 5.2. Firms in an intermediate situation are gathered in groups 2.2, 2.3, 3.2, 3.3, 3.4, 4.2, 4.3 and 4.4.

\section{Retention Factor}

Fig. 2 shows the projection of firms by means of CMLHL and according to the retention factor.

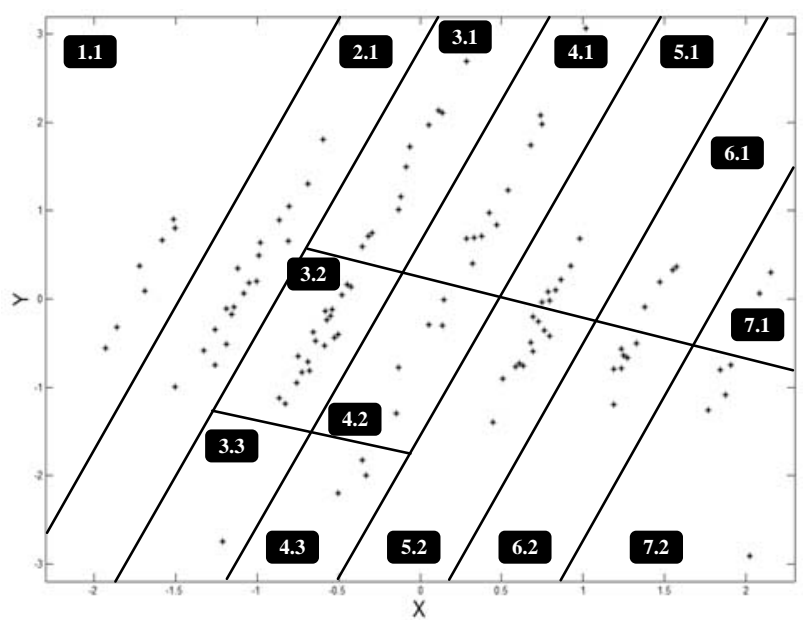

Fig. 2. CMLHL projection of the Retention Factor dataset.

The first feature to be considered for this factor (Candidates are selected according to their fitting with the firm) is very important, and causes a vertical order of the data. Those firms at the top of Fig. 2 are the ones with the highest values for this feature, while the ones at the bottom are those whose fitting to new employees is almost inexistent. Two of the companies do not take this issue into account at all and are depicted at the very bottom of the projection.

Regarding the second feature, the projection shows a horizontal distribution of the data. Most of the companies are in an intermediate situation, as only six of them take the highest value (in the right side of Fig. 2) and seven of them take the lowest value (in the left side of Fig. 2). This feature is related with the previous one as there is a coincidence between the global fitting and the firm culture.

The third one of the retention features reveals a precise tendency in the projection. Firms at the bottom of Fig. 2 are the ones with highest values for this feature. The lowest values place firms at the top of the projection. There is not a big amount of firms taking the maximum value for this feature. On the contrary, most of the firms take medium/minimum values. This can be identified as a deficiency in the important task of new employee integration. Considering the previous features, those firms that get the highest value in the employee selection according to the global fitting with the company do not care about the successful integration of new employees.

According to the fourth feature, the firms are ordered in a similar way as for the second one (highest scores in the right side and the lowest ones in the left side). This 
is coherent as the sponsoring of social activities for employees is part of the culture values of the company and leads to a high level of commitment that may be extended to other cultural components. The fifth variable shows a vertical tendency in the data ordering as in the case of the first feature related with the acquisition.

From a general perspective, the whole dataset is split in fourteen different groups according to the retention factor. The best practices are performed by the firms in groups 6.1 and 7.1 (Fig. 2). On the other hand, firms in group 1.1 are those in the worst situation regarding the analyzed factor. Some other firms are in an intermediate situation and are located in groups 3.1, 3.2, 4.1, 4.2, 5.1 and 5.2 (Fig. 2).

\subsection{Comparative Study}

To compare the CMLHL projections with those obtained from some other unsupervised techniques, Principal Component Analysis (PCA) [17], MLHL and SelfOrganizing Map (SOM) [23] have been also applied to the HR dataset. The obtained projections are shown in Fig. 3. The PCA neural version [24], MLHL and CMLHL are compared below as the three of them are projection models based on unsupervised learning, aimed to provide a visual analysis of the internal structure of a data set.

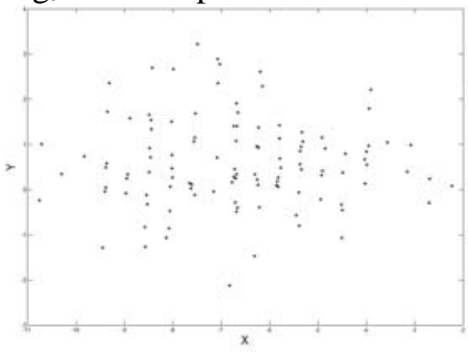

a) PCA projection of acquisition factor.

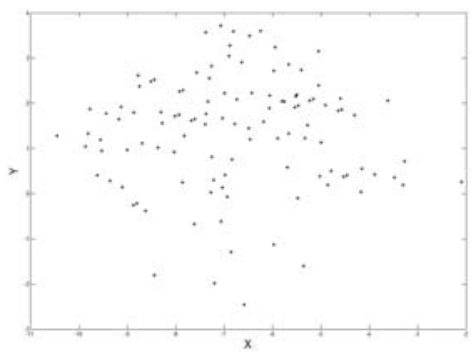

c) PCA projection of retention factor.

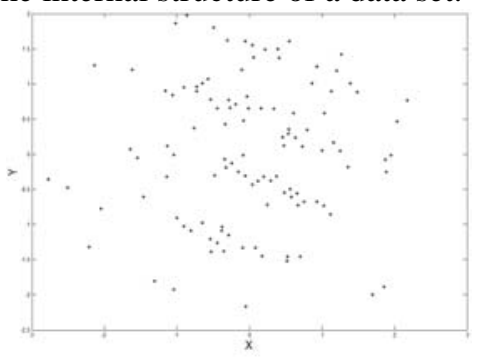

b) MLHL projection of acquisition factor.

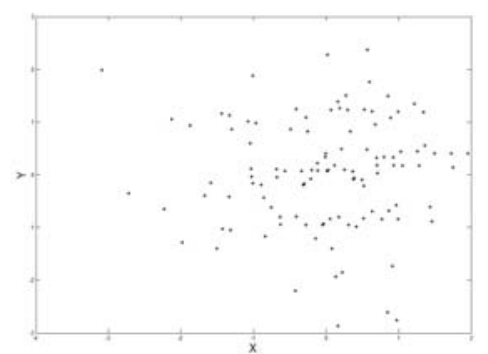

d) MLHL projection of retention factor.

Fig. 3. PCA and MLHL projections of HR data (acquisition and retention datasets).

The sum of the data variance accumulated by the two first principal components is $67.8 \%$ for the acquisition factor (Fig. 3.a) and $66.9 \%$ for the retention factor (Fig. 3.c). As can be seen in Fig. 3 none of them reveals the inner structure of the data for 
any of the analyzed factors. On the other hand, the MLHL projections of both acquisition (Fig. 3.b) and retention (Fig. 3.d) factors allow a deeper analysis of the data.

Regarding the acquisition factor, MLHL provides a less precise visualization of the data structure than CMLHL. However, a certain organization of the data can be identified. Firms in the left side of the projection (Fig. 3.b) take high values concerning the second, third and fifth features. Firms in the right side of the projection are in the opposite situation regarding the mentioned features. It is the other way round considering the first and fourth features. Firms in the centre of the projection (Fig. 3.b) take intermediate values, whose situation improve if they are at the bottom of the projection.

An in-deep analysis of the retention factor and the MLHL projection (Fig. 3.d), shows that firms in the left side of the projection take the highest values regarding the second, third and fourth features. Firms taking the highest values for the first and fifth features are located in the right side of the projection.

On the other hand, SOM has also been applied to the above described dataset. For the sake of brevity, graphical results of the SOM mapping have not been included in this study. It can be said that the SOM does not provides with a clear clustering of the data, as neurons do not collectively respond to a certain kind of input data.

While previous research [25] [26] [27] outlines the effectiveness of HRM practices (such as selective hiring, performance appraisal, self-managed team, extensive training and some others) to increase the firm's levels of knowledge acquisition, sharing and application, some other studies [5] [28] [29] reach evidence that KM capabilities play a mediating role between HRM practices and overall firm performance.

\section{Conclusions and Future Work}

The main target of the present study is to provide a powerful and robust (to be applied to disparate data) intelligent tool for researching on KM and HR, from purely theoretical formulations to applications allowing a diagnosis based on the captured data.

This objective has been fulfilled as CMLHL has been able to visualize the data in an ordered way, leading to a fruitful analysis of the firms' situation. The clear-cut grouping in the projections allows the definition of firms according to the acquisition and retention settings. Subsequent corrective actions can be proposed according to the firm strategy.

In conclusion, the application of unsupervised neural techniques has contributed to successfully detect the HR practices in firms and how to evolve and progress toward a more efficient deployment and execution of them. This will greatly contribute to improve the efficiency and competitiveness of firms. Thus, CMLHL provides with a strategic viewpoint that allows us to identify the specific key characteristics for companies to improve their knowledge management status. 
Future work will focus on extending this study with some other factors that are important in the HR and KM fields (described in Section 2), and in the application of other unsupervised models such as the ViSOM [30].

Acknowledgments. This research has been partially supported through the Spanish Ministry of Science and Innovation (MICINN) under projects DPI2010-16920, Consolider CSD2010-00034 and CIT-020000-2009-12 (funded by the European Regional Development Fund); project of the Spanish Ministry of Science and Innovation TIN2010-21272-C02-01 (funded by the European Regional Development Fund). The authors would also like to thank the vehicle interior manufacturer, Grupo Antolin Ingenieria S.A., within the framework of the MAGNO2008 - 1028.- CENIT Project also funded by the MICINN.

\section{References}

1. Sivan, Y.Y.: Nine Keys to a Knowledge Infrastructure: A Proposed Analytic Framework for Organizational Knowledge Management. In: WebNet 2000 - World Conference on the WWW and Internet. pp. 495-500. AACE (2000)

2. Shu-Mei, T.: The Effects of Information Technology on Knowledge Management Systems. Expert Systems with Applications: An International Journal 35(1-2), 150-160 (2008)

3. Russell, S.J., Norvig, P.: Artificial Intelligence: a Modern Approach. Prentice Hall, Englewood Cliffs, NJ (1995)

4. Herrero, Á., Corchado, E., Sáiz, L., Abraham, A.: DIPKIP: A Connectionist Knowledge Management System to Identify Knowledge Deficits in Practical Cases. Computational Intelligence 26(1), 26-56 (2010)

5. Sáiz, L., Pérez, A.: Formación de las Capacidades de Creación de Conocimiento y Flexibilidad Organizativa en Empresas de Alta Tecnología. In: 4th International Conference on Industrial Engineering and Industrial Management. pp. (2010)

6. Spanish National Institute of Statistics. http://www.ine.es/

7. Soo, C.W., Devinney, T.M., Midgley, D.F.: External Knowledge Acquisition, Creativity and Learning in Organisational Problem Solving. International Journal of Technology Management 38(1), 137-159 (2007)

8. Akgün, A.E., Keskin, H., Byrne, J.C., Aren, S.: Emotional and Learning Capability and their Impact on Product Innovativeness and Firm Performance. Technovation 27(9), 501-513 (2007)

9. Maranto-Vargas, D., Rangel, R.G.T.: Development of Internal Resources and Capabilities as Sources of Differentiation of SME under Increased Global Competition: A Field Study in Mexico. Technol. Forecast. Soc. Chang. 74(1), 90-99 (2007)

10. Smith, K.G., Collins, C.J., Clark, K.D.: Existing Knowledge, Knowledge Creation Capability, and the Rate of New Product Introduction in High-Technology Firms. Academy of Management Journal 48, 346-357 (2005)

11. Grant, R.M.: Toward a Knowledge-based Theory of the Firm. Strategic Management Journal 17(10), 109-122 (1996)

12. Kamoche, K.N.: Managing People in Turbulent Economic Times: A Knowledge-creation and Appropriation Perspective. Asia Pacific Journal of Human Resources 44(1), 25 (2006)

13. Nonaka, I., Takeuchi, H.: The Knowledge-Creating Company. Oxford Press (1995)

14. Collins, C.J.: Strategic Human Resource Management and Knowledge-creation Capability: Examining the Black Box between HR and Firm Performance. PhD Thesis. Robert H. Smith School of Business. University of Maryland. (2000) 
15. Dolan, S.L., Mach, M., Olivera, V.S.: HR Contribution to a Firm's Success Examined from a Configurational Perspective: An Exploratory Study Based on the Spanish CRANET Data. Management Review 16(2), 272 (2005)

16. Hotelling, H.: Analysis of a Complex of Statistical Variables into Principal Components. Journal of Education Psychology 24, 417-444 (1933)

17. Pearson, K.: On Lines and Planes of Closest Fit to Systems of Points in Space. Philosophical Magazine 2(6), 559-572 (1901)

18. Friedman, J.H., Tukey, J.W.: A Projection Pursuit Algorithm for Exploratory DataAnalysis. IEEE Transactions on Computers 23(9), 881-890 (1974)

19. Corchado, E., MacDonald, D., Fyfe, C.: Maximum and Minimum Likelihood Hebbian Learning for Exploratory Projection Pursuit. Data Mining and Knowledge Discovery 8(3), 203-225 (2004)

20. Corchado, E., Han, Y., Fyfe, C.: Structuring Global Responses of Local Filters Using Lateral Connections. Journal of Experimental \& Theoretical Artificial Intelligence 15(4), 473-487 (2003)

21. Corchado, E., Fyfe, C.: Connectionist Techniques for the Identification and Suppression of Interfering Underlying Factors. International Journal of Pattern Recognition and Artificial Intelligence 17(8), 1447-1466 (2003)

22. Seung, H.S., Socci, N.D., Lee, D.: The Rectified Gaussian Distribution. Advances in Neural Information Processing Systems 10, 350-356 (1998)

23. Kohonen, T.: The Self-Organizing Map. Proceedings of the IEEE 78(9), 1464-1480 (1990)

24. Fyfe, C.: A Neural Network for PCA and Beyond. Neural Processing Letters 6(1-2), 33-41 (1997)

25. Har, W.C., In, T.B., Phaik, L.S., Hsien, L.V.: The Impact of HRM Practices on KM: A Conceptual Model. Journal of Applied Sciences Research 6(12), 6281-6291 (2010)

26. Zheng, W., Yang, B., McLean, G.N.: Linking Organizational Culture, Structure, Strategy, and Organizational Effectiveness: Mediating Role of Knowledge Management. Journal of Business Research 63(7), 763-771 (2010)

27. Liao, H., Toya, K., Lepak, D.P., Hong, Y.: Do They See Eye to Eye? Management and Employee Perspectives of High-Performance Work Systems and Influence Processes on Service Quality. Journal of Applied Psychology 94(2), 371-391 (2009)

28. Chen, C.-J., Huang, J.-W.: Strategic Human Resource Practices and Innovation Performance - The Mediating Role of Knowledge Management Capacity. Journal of Business Research 62(1), 104-114 (2009)

29. Theriou, G.N., Chatzoglou, P.D.: Enhancing Performance through Best HRM Practices, Organizational Learning and Knowledge Management: A Conceptual Framework. European Business Review 20(3), 185-207 (2008)

30. Yin, H.: ViSOM - a Novel Method for Multivariate Data Projection and Structure Visualization. IEEE Transactions on Neural Networks 13(1), 237-243 (2002) 